Supporting Information for

\title{
Mesoporous Bimetallic Au@Rh Core-Shell Nanowires as Efficient Electrocatalysts for pH-Universal Hydrogen Evolution
}

Hongjing Wang, Shiqian Jiao, Songliang Liu, Shengqi Wang, Tongqing Zhou, You Xu, Xiaonian Li, Ziqiang Wang, * and Liang Wang*

State Key Laboratory Breeding Base of Green-Chemical Synthesis Technology, College of Chemical Engineering, Zhejiang University of Technology, Hangzhou 310014, P. R. China.

Corresponding authors’ E-mails: zqwang@zjut.edu.cn; wangliang@zjut.edu.cn 

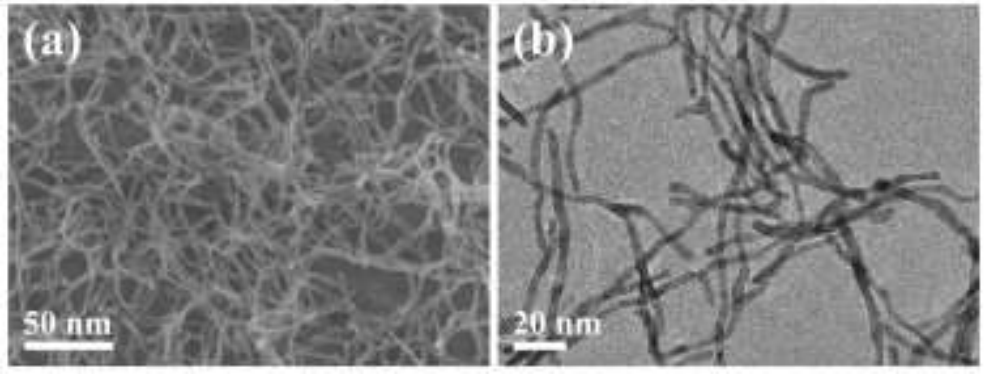

Figure S1. (a) SEM and (b) TEM images of Au NWs.

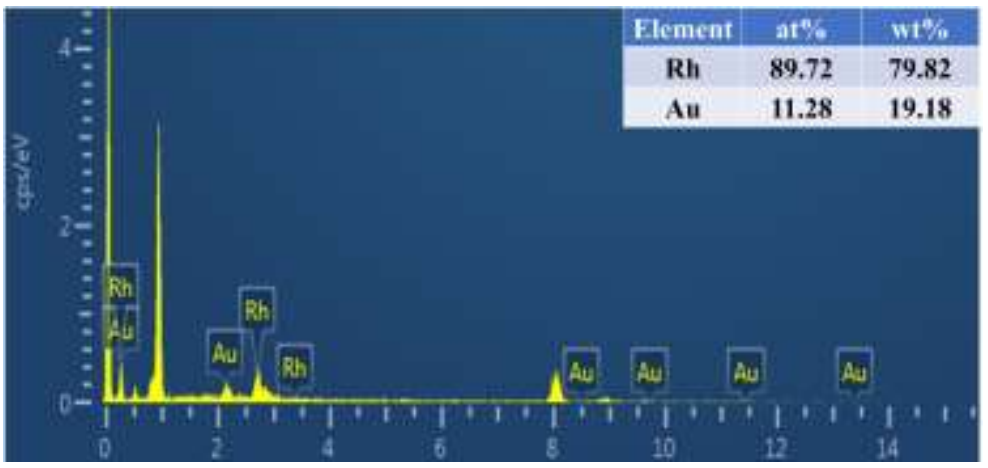

Figure S2. EDX spectrum of the Au@mRh NWs.
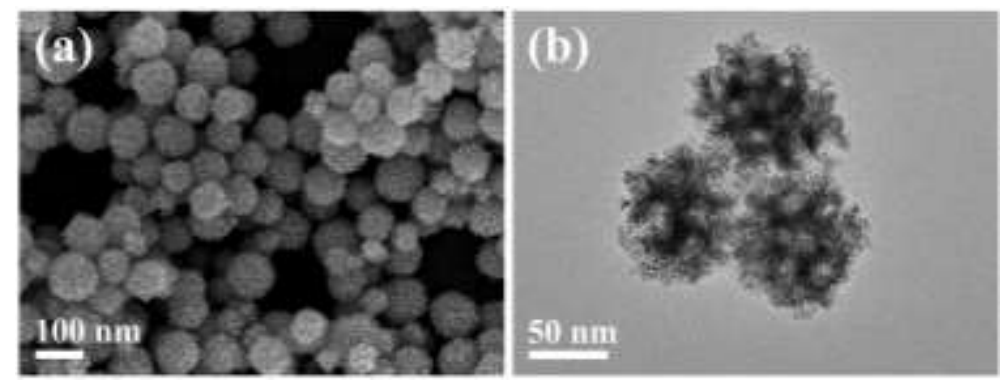

Figure S3. (a) SEM and (b) TEM images of mRh NSs. 


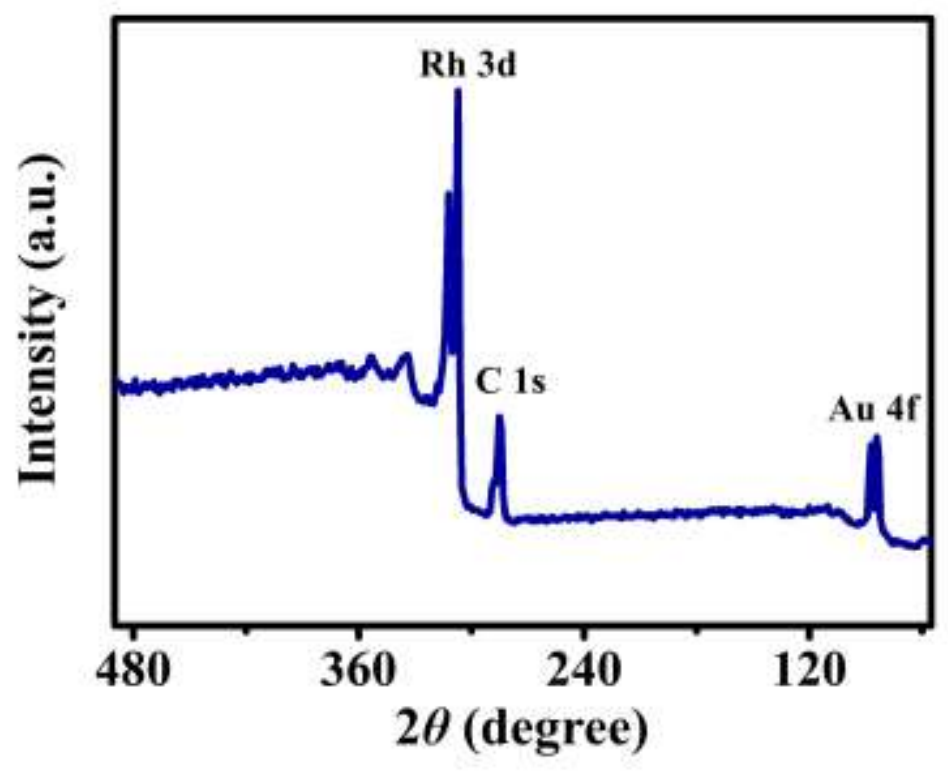

Figure S4. XPS survery spectrum of the Au@mRh NWs.

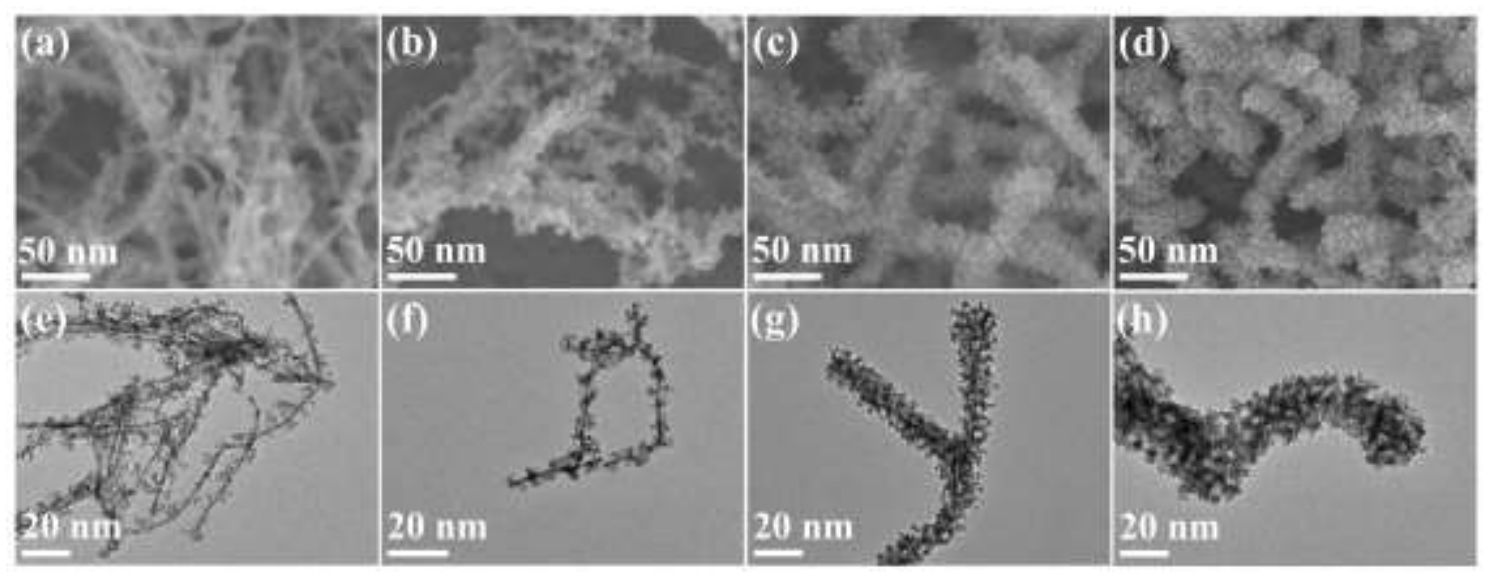

Figure S5. Typical SEM (a-d) and TEM (e-h) images of Au@mRh NWs obtained at different reaction times in the typical synthetic conditions: (a, e) 4 h, (b, f) 8 h, (c, g) 12 h, (d, h) 16 h. 

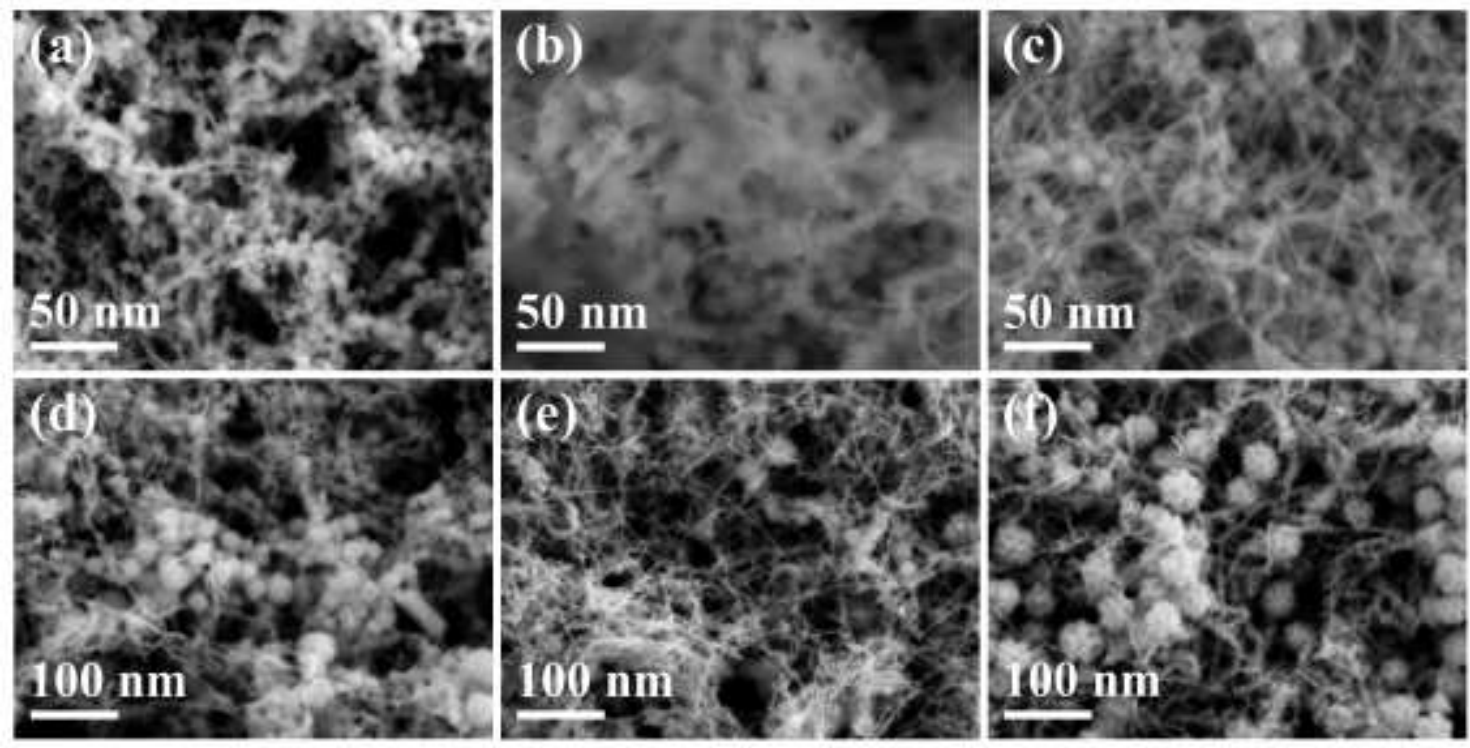

Figure S6. (a) SEM image of the sample prepared without PEO- $b$-PMMA, SEM images of samples prepared under the typical conditions by replacing PEO- $b$-PMMA with (b) PS- $b$-PEO, (c) PVP, (d) CTAC, (e) DM970, and (f) F127.
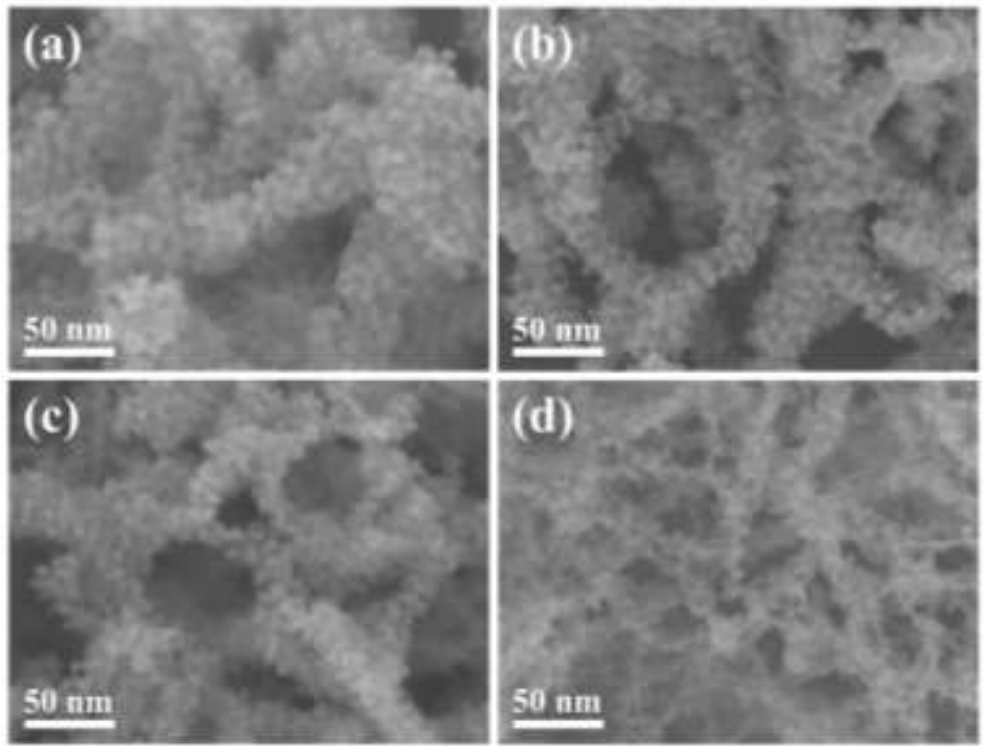

Figure S7. SEM images for the samples with different adding amount of Au NWs in the typical synthetic conditions: (a) $25 \mu \mathrm{L}$, (b) $75 \mu \mathrm{L}$, (c) $100 \mu \mathrm{L}$, and (d) $150 \mu \mathrm{L}$. 



Figure S8. (a) The polarization curves of samples with different $\mathrm{Au} / \mathrm{Rh}$ ratios in $0.5 \mathrm{M} \mathrm{H}_{2} \mathrm{SO}_{4}$ solution and (b) the overpotentials of various catalysts at $10 \mathrm{~mA} \mathrm{~cm}^{-2}$.



Figure S9. The hydrogen production for different electrocatalysts. 



Figure S10. CO stripping curves of various catalysts in acid electrolytes.



Figure S11. The $\mathrm{N}_{2}$ adsorption-desorption isotherm along with the pore size distribution curve for the Au@mRh NWs. 



Figure S12. Nyquist plots for different catalysts recorded in (a) $0.5 \mathrm{M} \mathrm{H}_{2} \mathrm{SO}_{4}$, (b) $1.0 \mathrm{M} \mathrm{KOH}$ and (c) 1.0 M PBS. 
Table S1. Comparison of HER activities in $0.5 \mathrm{M} \mathrm{H}_{2} \mathrm{SO}_{4}$ for $\mathrm{Au} @ \mathrm{mRh} \mathrm{NWs}$ with other reported Rh-based electrocatalysts.

\begin{tabular}{|c|c|c|c|c|}
\hline Catalysts & Electrolyte & $\begin{array}{l}\text { Overpotential at } \\
10 \mathrm{~mA} \mathrm{~cm}^{-2}(\mathrm{mV})\end{array}$ & $\begin{array}{l}\text { Tafel slope } \\
\left(\mathrm{mV} \operatorname{dec}^{-1}\right)\end{array}$ & Ref. \\
\hline Au@mRh NWs & $0.5 \mathrm{M} \mathrm{H}_{2} \mathrm{SO}_{4}$ & 30 & 29 & This work \\
\hline $\begin{array}{c}\mathrm{Rh}-\mathrm{Au}-\mathrm{Si} \\
\text { nanocomposite }\end{array}$ & $0.5 \mathrm{M} \mathrm{H}_{2} \mathrm{SO}_{4}$ & 60 & 24 & 1 \\
\hline $\begin{array}{l}\text { Rh-Ag-Si ternary } \\
\text { composites }\end{array}$ & $0.5 \mathrm{M} \mathrm{H}_{2} \mathrm{SO}_{4}$ & 120 & 51 & 2 \\
\hline $\mathrm{rGO} / \mathrm{CoP}-\mathrm{Rh}$ catalysts & $0.5 \mathrm{M} \mathrm{H}_{2} \mathrm{SO}_{4}$ & 72 & 43 & 3 \\
\hline $\begin{array}{c}\mathrm{Rh}_{2} \mathrm{~S}_{3} \text { hexagonal } \\
\text { nanoprisms }\end{array}$ & $0.5 \mathrm{M} \mathrm{H}_{2} \mathrm{SO}_{4}$ & 117 & 44 & 4 \\
\hline $\begin{array}{c}\mathrm{Rh}-\mathrm{MoS}_{2} \\
\text { nanocomposites }\end{array}$ & $0.5 \mathrm{M} \mathrm{H}_{2} \mathrm{SO}_{4}$ & 47 & 24 & 5 \\
\hline $\mathrm{Rh} / \mathrm{F}$-graphene & $0.5 \mathrm{M} \mathrm{H}_{2} \mathrm{SO}_{4}$ & 46 & 30 & 6 \\
\hline $\mathrm{Au}_{75} \mathrm{Rh}_{25} / \mathrm{C}$ & $0.5 \mathrm{M} \mathrm{H}_{2} \mathrm{SO}_{4}$ & 64.1 & 33.8 & 7 \\
\hline Rh@CTF-1 & $0.5 \mathrm{M} \mathrm{H}_{2} \mathrm{SO}_{4}$ & 58 & 37 & 8 \\
\hline $\mathrm{Rh}-\mathrm{MoS}_{2}-4.8$ & $0.5 \mathrm{M} \mathrm{H}_{2} \mathrm{SO}_{4}$ & 65 & 54 & 9 \\
\hline
\end{tabular}


Table S2. Comparison of HER activities in 1.0 M KOH for $\mathrm{Au} @ \mathrm{mRh} \mathrm{NWs}$ with other reported Rh-based electrocatalysts.

\begin{tabular}{|c|c|c|c|c|}
\hline Catalysts & Electrolyte & $\begin{array}{l}\text { Overpotential at } \\
10 \mathrm{~mA} \mathrm{~cm}^{-2}(\mathrm{mV})\end{array}$ & $\begin{array}{l}\text { Tafel slope } \\
\left.(\mathrm{mV} \mathrm{dec})^{-1}\right)\end{array}$ & Ref. \\
\hline Au@mRh NWs & $1.0 \mathrm{M} \mathrm{КОН}$ & 32 & 33 & This work \\
\hline $\mathrm{Rh}-\mathrm{Rh}_{2} \mathrm{O}_{3}-\mathrm{NPs} / \mathrm{C}$ & $1.0 \mathrm{M} \mathrm{KOH}$ & 63 & 70 & 10 \\
\hline $\mathrm{Rh} / \mathrm{SWNTs}$ & $1.0 \mathrm{M} \mathrm{KOH}$ & 48 & 27 & 11 \\
\hline $\mathrm{Rh}-\mathrm{MoSe}_{2}$ & $1.0 \mathrm{M} \mathrm{KOH}$ & 73 & 118 & 12 \\
\hline Rh NSs & $1.0 \mathrm{M} \mathrm{KOH}$ & 43 & 107 & 13 \\
\hline RhCo-ANAs & $1.0 \mathrm{M} \mathrm{KOH}$ & 32.4 & 31.9 & 14 \\
\hline 4.2 Rh-CN & $1.0 \mathrm{M} \mathrm{KOH}$ & 46 & 42 & 15 \\
\hline $\mathrm{Rh}_{2} \mathrm{~S}_{3} / \mathrm{NC}$ & $1.0 \mathrm{M} \mathrm{KOH}$ & 38 & 41 & 16 \\
\hline PtRh NAA & $1.0 \mathrm{M} \mathrm{KOH}$ & 55 & 45 & 17 \\
\hline RhPd-H & $1.0 \mathrm{M} \mathrm{KOH}$ & 40 & 35.7 & 18 \\
\hline
\end{tabular}


Table S3. Comparison of HER activities in 1.0 M PBS for $\mathrm{Au} @ \mathrm{mRh}$ NWs with other reported electrocatalysts.

\begin{tabular}{|c|c|c|c|c|}
\hline Catalysts & Electrolyte & $\begin{array}{l}\text { Overpotential at } \\
10 \mathrm{~mA} \mathrm{~cm}^{-2}(\mathrm{mV})\end{array}$ & $\begin{array}{l}\text { Tafel slope } \\
\left.(\mathrm{mV} \mathrm{dec})^{-1}\right)\end{array}$ & Ref. \\
\hline Au@mRh NWs & 1.0 M PBS & 36 & 61 & This work \\
\hline $\mathrm{Au}-\mathrm{Rh}$ aerogel & 1.0 M PBS & 18 & 34 & 19 \\
\hline RhCo NSs & 1.0 M PBS & 31 & 34 & 14 \\
\hline $\mathrm{Rh}_{2} \mathrm{P} @ \mathrm{NC}$ & 1.0 M PBS & 46 & 37 & 20 \\
\hline Ru-Co NPs@N-C & 1.0 M PBS & 55 & 82 & 21 \\
\hline Pt@NOMC-A & 1.0 M PBS & 65 & 70 & 22 \\
\hline Pt-Pd@NPA & $1.0 \mathrm{M}$ PBS & 34.8 & 32.2 & 23 \\
\hline $\mathrm{PdP}_{2} / \mathrm{CB}$ & $1.0 \mathrm{M}$ PBS & 84.6 & 72.3 & 24 \\
\hline
\end{tabular}




\section{References}

(1) Jiang, B.; Yang, L.; Liao, F.; Sheng, M.; Zhao, H.; Lin, H.; Shao, M. A Stepwise-Designed Rh-Au-Si Nanocomposite that Surpasses Pt/C Hydrogen Evolution Activity at High Overpotentials. Nano Res. 2017, 10 (5), 1749-1755, DOI: 10.1007/s12274-017-1447-0.

(2) Jiang, B.; Sun, Y.; Liao, F.; Shen, W.; Lin, H.; Wang, H.; Shao, M. Rh-Ag-Si Ternary Composites: Highly Active Hydrogen Evolution Electrocatalysts over Pt-Ag-Si. J. Mater. Chem. A 2017, 5 (4), 1623-1628, DOI: 10.1039/c6ta09619k.

(3) Zheng, H.; Huang, X.; Gao, H.; Dong, W.; Lu, G.; Chen, X.; Wang, G. Decorating Cobalt Phosphide and Rhodium on Reduced Graphene Oxide for High-Efficiency Hydrogen Evolution Reaction. J. Energy Chem. 2019, 34, 72-79, DOI: 10.1016/j.jechem.2018.10.009.

(4) Yoon, D.; Seo, B.; Lee, J.; Nam, K. S.; Kim, B.; Park, S.; Baik, H.; Hoon Joo, S.; Lee, K. Facet-Controlled Hollow $\mathrm{Rh}_{2} \mathrm{~S}_{3}$ Hexagonal Nanoprisms as Highly Active and Structurally Robust Catalysts toward Hydrogen Evolution Reaction. Energy Environ. Sci. 2016, 9 (3), 850-856, DOI: 10.1039/c5ee03456f.

(5) Cheng, Y.; Lu, S.; Liao, F.; Liu, L.; Li, Y.; Shao, M. Rh-MoS 2 Nanocomposite Catalysts with Pt-Like Activity for Hydrogen Evolution Reaction. Adv. Funct. Mater. 2017, 27 (23), 1700359, DOI: 10.1002/adfm.201700359.

(6) Shen, W.; Ge, L.; Sun, Y.; Liao, F.; Xu, L.; Dang, Q.; Kang, Z.; Shao, M. Rhodium Nanoparticles/F-Doped Graphene Composites as Multifunctional Electrocatalyst Superior to Pt/C for Hydrogen Evolution and Formic Acid Oxidation Reaction. ACS Appl. Mater. Interfaces 2018, 10 (39), 33153-33161, DOI: 10.1021/acsami.8b09297.

(7) Bian, T.; Xiao, B.; Sun, B.; Huang, L.; Su, S.; Jiang, Y.; Xiao, J.; Yuan, A.; Zhang, H.; Yang, D. Local Epitaxial Growth of Au-Rh Core-Shell Star-Shaped Decahedra: A Case for Studying 
Electronic and Ensemble Effects in Hydrogen Evolution Reaction. Appl. Catal., B 2020, 263, DOI: 10.1016/j.apcatb.2019.118255.

(8) Siebels, M.; Schlüsener, C.; Thomas, J.; Xiao, Y.-X.; Yang, X.-Y.; Janiak, C. Rhodium Nanoparticles Supported on Covalent Triazine-Based Frameworks as Re-Usable Catalyst for Benzene Hydrogenation and Hydrogen Evolution Reaction. J. Mater. Chem. A 2019, 7 (19), 11934-11943, DOI: 10.1039/c8ta12353e.

(9) Meng, X.; Ma, C.; Jiang, L.; Si, R.; Meng, X.; Tu, Y.; Yu, L.; Bao, X.; Deng, D. Distance Synergy of $\mathrm{MoS}_{2}$-Confined Rhodium Atoms for Highly Efficient Hydrogen Evolution. Angew. Chem., Int. Ed. 2020, 59 (26), 10502-10507, DOI: 10.1002/anie.202003484.

(10) Kundu, M. K.; Mishra, R.; Bhowmik, T.; Barman, S. Rhodium Metal-Rhodium Oxide $\left(\mathrm{Rh}-\mathrm{Rh}_{2} \mathrm{O}_{3}\right)$ Nanostructures with Pt-Like or Better Activity towards Hydrogen Evolution and Oxidation Reactions (HER, HOR) in Acid and Base: Correlating its HOR/HER Activity with Hydrogen Binding Energy and Oxophilicity of the Catalyst. J. Mater. Chem. A 2018, 6 (46), 23531-23541, DOI: 10.1039/c8ta07028h.

(11) Zhang, W.; Zhang, X.; Chen, L.; Dai, J.; Ding, Y.; Ji, L.; Zhao, J.; Yan, M.; Yang, F.; Chang, C.-R.; Guo, S. Single-Walled Carbon Nanotube Induced Optimized Electron Polarization of Rhodium Nanocrystals To Develop an Interface Catalyst for Highly Efficient Electrocatalysis. ACS Catal. 2018, 8 (9), 8092-8099, DOI: 10.1021/acscatal.8b02016.

(12) Zhao, Y.; Yang, C.; Mao, G.; Su, J.; Cheng, G.; Luo, W. Ultrafine Rh Nanoparticle Decorated $\mathrm{MoSe}_{2}$ Nanoflowers for Efficient Alkaline Hydrogen Evolution Reaction. Inorg. Chem. Front. 2018, 5 (11), 2978-2984, DOI: 10.1039/c8qi00874d.

(13) Zhang, N.; Shao, Q.; Pi, Y.; Guo, J.; Huang, X. Solvent-Mediated Shape Tuning of Well-Defined Rhodium Nanocrystals for Efficient Electrochemical Water Splitting. Chem. Mater. 
2017, 29 (11), 5009-5015, DOI: 10.1021/acs.chemmater.7b01588.

(14) Zhao, Y.; Bai, J.; Wu, X.-R.; Chen, P.; Jin, P.-J.; Yao, H.-C.; Chen, Y. Atomically Ultrathin RhCo Alloy Nanosheet Aggregates for Efficient Water Electrolysis in Broad pH Range. J. Mater. Chem. A 2019, 7 (27), 16437-16446, DOI: 10.1039/c9ta05334d.

(15) Jiang, B.; Huang, A.; Wang, T.; Shao, Q.; Zhu, W.; Liao, F.; Cheng, Y.; Shao, M. Rhodium/Graphitic-Carbon-Nitride Composite Electrocatalyst Facilitates Efficient Hydrogen Evolution in Acidic and Alkaline Electrolytes. J. Colloid Interface Sci. 2020, 571, 30-37, DOI: 10.1016/j.jcis.2020.03.022.

(16) Zhang, C.; Liu, H.; Liu, Y.; Liu, X.; Mi, Y.; Guo, R.; Sun, J.; Bao, H.; He, J.; Qiu, Y.; Ren, J.; Yang, X.; Luo, J.; Hu, G. $\mathrm{Rh}_{2} \mathrm{~S}_{3} / \mathrm{N}$-Doped Carbon Hybrids as pH-Universal Bifunctional Electrocatalysts for Energy-Saving Hydrogen Evolution. Small Methods 2020, 2000208, DOI: 10.1002/smtd.202000208.

(17) Jin, Y.; Chen, F.; Wang, J.; Guo, L.; Jin, T.; Liu, H. Lamellar Platinum-Rhodium Aerogels with Superior Electrocatalytic Performance for Both Hydrogen Oxidation and Evolution Reaction in Alkaline Environment. J. Power Sources 2019, 435, DOI: 10.1016/j.jpowsour.2019.226798.

(18) Fan, J.; Wu, J.; Cui, X.; Gu, L.; Zhang, Q.; Meng, F.; Lei, B.-H.; Singh, D. J.; Zheng, W. Hydrogen Stabilized RhPdH 2D Bimetallene Nanosheets for Efficient Alkaline Hydrogen Evolution. J. Am. Chem. Soc. 2020, 142 (7), 3645-3651, DOI: 10.1021/jacs.0c00218.

(19) Du, R.; Jin, W.; Hübner, R.; Zhou, L.; Hu, Y.; Eychmüller, A. Engineering Multimetallic Aerogels for pH-Universal HER and ORR Electrocatalysis. Adv. Energy Mater. 2020, 10 (12), DOI: 10.1002/aenm.201903857.

(20) Pu, Z.; Amiinu, I. S.; He, D.; Wang, M.; Li, G.; Mu, S. Activating Rhodium Phosphide-Based Catalysts for the pH-Universal Hydrogen Evolution Reaction. Nanoscale 2018, 10 (26), 
12407-12412, DOI: 10.1039/c8nr02854k.

(21) Yuan, S.; Pu, Z.; Zhou, H.; Yu, J.; Amiinu, I. S.; Zhu, J.; Liang, Q.; Yang, J.; He, D.; Hu, Z.; Van Tendeloo, G.; Mu, S. A Universal Synthesis Strategy for Single Atom Dispersed Cobalt/Metal Clusters Heterostructure Boosting Hydrogen Evolution Catalysis at all pH Values. Nano Energy 2019, 59, 472-480, DOI: 10.1016/j.nanoen.2019.02.062.

(22) Yin, Y.; Liu, T.; Liu, D.; Wang, Z.; Deng, Q.; Qu, D.; Xie, Z.; Tang, H.; Li, J. Confining Nano-Sized Platinum in Nitrogen Doped Ordered Mesoporous Carbon: An Effective Approach toward Efficient and Robust Hydrogen Evolution Electrocatalyst. J. Colloid Interface Sci. 2018, 530, 595-602, DOI: 10.1016/j.jcis.2018.06.096.

(23) Yang, C.; Lei, H.; Zhou, W. Z.; Zeng, J. R.; Zhang, Q. B.; Hua, Y. X.; Xu, C. Y. Engineering Nanoporous Ag/Pd Core/Shell Interfaces with Ultrathin Pt Doping for Efficient Hydrogen Evolution Reaction over a Wide pH Range. J. Mater. Chem. A 2018, 6 (29), 14281-14290, DOI: 10.1039/c8ta04059a.

(24) Luo, F.; Zhang, Q.; Yu, X.; Xiao, S.; Ling, Y.; Hu, H.; Guo, L.; Yang, Z.; Huang, L.; Cai, W.; Cheng, H. Palladium Phosphide as a Stable and Efficient Electrocatalyst for Overall Water Splitting. Angew. Chem., Int. Ed. 2018, 57 (45), 14862-14867, DOI: 10.1002/anie.201810102. 